\title{
A SPECTROSCOPIC SEARCH FOR COLLIDING WINDS IN \\ A COMPLETE SAMPLE OF WC/WO STARS IN THE MAGELLANIC CLOUDS
}

\author{
P. BARTZAKOS and A.F.J. MOFFAT \\ Département de Physique, Université de Montréal, C.P. 6128, Succursale A, Montréal, \\ P.Q. H3C 3J7, Canada, and Observatoire du Mont-Mégantic \\ and \\ V.S. NIEMELA \\ Instituto de Astronomia y Fisica del Espacio, Buenos Aires, Argentina
}

\begin{abstract}
The Magellanic Clouds provide an ideal laboratory to study the properties of Wolf-Rayet stars. In particular, the $25 \mathrm{WC} / \mathrm{WO}$ stars that they contain are nearly all of the same sub-class (21 WC4, one WC5, one WC6 and one WO in the Large Magellanic Cloud; one WO in the Small Magellanic Cloud). This, along with their formation in the same isolated environment, suggests that the wind structure is similar in each star. Thus, a study of other characteristics, such as the effects of binary separation and period on binary wind collisions, can be undertaken with little concern for differing winds. Some 30 high-quality CCD spectra for each of the 25 stars were obtained during three observing missions in the past decade. Although the analysis is not yet complete, the sample clearly contains four large-amplitude $\mathrm{WR}+\mathrm{O}$ binaries with periods ranging from two to seventeen days and possibly other long-perioc systems. This paper deals with the wind interaction of the star B22.
\end{abstract}

Key words: stars: Wolf-Rayet - binaries - winds - Magellanic Clouds

\section{Objectives and data sample}

This project comprises a spectroscopic survey of all WC/WO-class stars in the Magellanic Clouds. Its original objective in 1984 was to study the binary frequency of these stars, which were formed in a different environment than that of the Galaxy. As well, the project included a study of stellar masses in those binaries found. With the subsequent realisation that the strong stellar winds of a Wolf-Rayet star and of its companion would collide and produce shocks, this study was extended to include the wind collisions of the appropriate binaries found.

The Clouds provide an excellent laboratory for such a study. All stars in each galaxy formed in the same isolated environment. Being nearly all of the same sub-class, they must have similar wind structure.

Of the twenty-four LMC WC/WO stars, twenty-one are of class WC4 with one of each of WC5, WC6 and WO class (Smith, Shara \& Moffat 1990). The only Small Magellanic Cloud WC/WO star is of type WO4. Most of the twenty-five stars were studied during three spectroscopy observing runs: thirteen nights in December of 1984 at CTIO, and eight nights in January 
1993 plus an additional sixteen nights in November of 1993 at the El Leoncito Observatory. The spectral region extended from approximately $5000 \AA$ to $6200 \AA$, with an inverse dispersion of about $120 \AA / \mathrm{mm}$.

In this progress report, the binary frequency of the sample and the spectacular wind collision of B22 in the Large Magellanic Cloud will be discussed. A previous study on the masses of the four most obvious binaries was reported by Moffat, Niemela \& Marraco (1990 MNM). Only the first two observing missions are included here; they do not include most of the fainter MG stars (Morgan \& Good 1985).

\section{Binary frequency}

Radial velocities of the stars were obtained from the Doppler shifts of the CIv $5802 / 5812 \AA$ doublet. Due to their broadness, the two lines are completely blended and so were treated as one line.

The only definite binary stars found were those that were previously reported as being binary (MNM). These were B22, B31 and B32 in the Large Magellanic Cloud and AB8 in the Small Magellanic Cloud.

The radial velocities of $\mathrm{B} 94$ for the observing run of January 1993 increased steadily by about $120 \mathrm{~km} / \mathrm{s}$ over eight days, which is about six times greater than the standard deviation of radial velocities about the mean for those stars that have no apparent orbital motion. This could be a long-period binary star but judgement awaits analysis of the data from the November 1993 observing mission. Niemela (these proceedings) also reported clear radial velocity variations of B44 over several years.

Thus, there are at least four binary systems and two likely long-period binaries among the sample. The apparent binary frequency of $\mathrm{MC} \mathrm{WC/WO}$ stars is close to twenty-five percent. This is considerably less than that for the Galaxy and the predictions of Maeder (1991). However, there might be other undetected long-period binaries in this sample.

\section{The behaviour of the carbon $5696 \AA$ line of $\mathrm{B22}$}

B22 is the only star that was classified as late as WC6 (MNM). This classification was deduced by comparing the relative line strengths of $\mathrm{CIV}_{\mathrm{IV}} 5802$ $-5812 \AA$ and $C_{I I I} 5696 \AA$. This assumed that both lines were formed in the wind of the WR star.

However, the $\mathrm{C}_{\text {III }}$ line does not follow the orbital motion defined by the CIv line (Fig. 1). The motion of this line appears sinusoidal, with the same period as that of the $\mathrm{C}_{\text {Iv }}$ line but with a large phase shift. The $\mathrm{C}_{\text {II }}$ line is thus not inherently formed in the WR wind and must be produced by some other process. The classification of the $\mathrm{WC}$ component of $\mathrm{B} 22$ should be revised from WC6 to WC4, making it more like the other WC stars. 


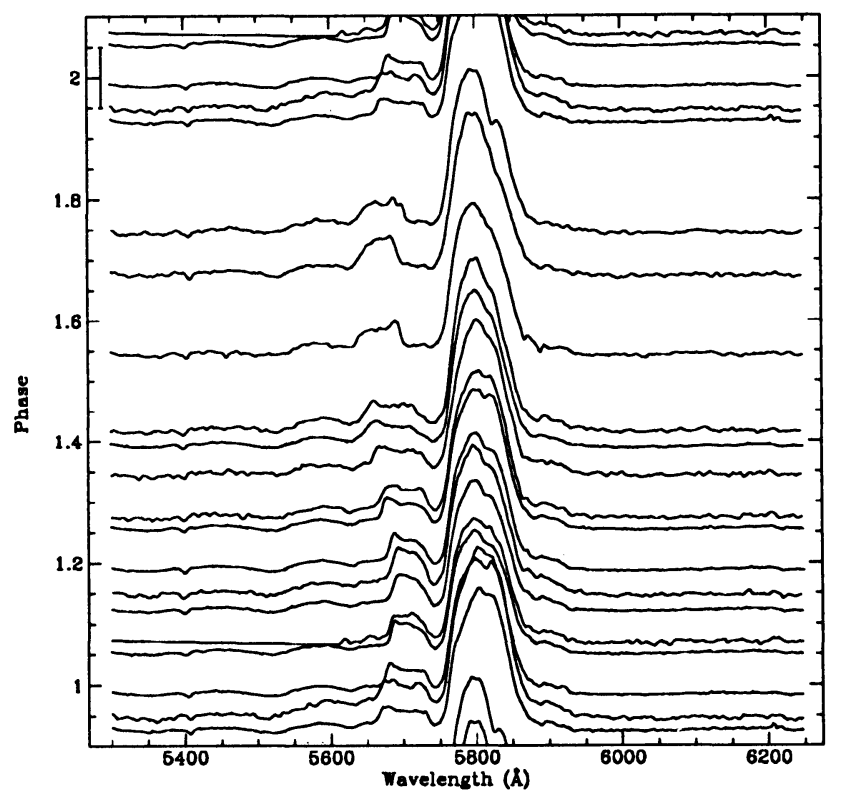

Fig. 1. Montage of spectra for B22. Error bar indicates continuum level.

The CIII line profile shape comprises a nearly rectangular base with a more or less clear peak at each of its red and blue extremes. This double-peaked profile changes throughout the orbital cycle; it is relatively narrow and high slightly after conjunction and is wider and flatter just after quadrature.

A rough model, inspired by Lührs (1991), can be used to explain the phenomenon. Both the WR star and the companion star are assumed to emit spherically symmetric winds. Colliding, they form a bow-shock that wraps around the companion. The material in the shock-cone region is greatly heated. As it flows outward along the shock, it cools, giving rise to various ions, including CIII at a certain point (assumed simply to comprise an optically thin band around the shock-cone).

The emission of such a band gives rise to a double-peaked profile, with the peaks at its extreme red and blue ends (Fig. 2). At quadrature, the profile is at its widest and flattest; the $\mathrm{C}_{\text {III }}$ band is viewed essentially from the side, so that the observer sees band material (centred at zero velocity) at the highest velocities of approach and of recession (for a fairly wide cone opening angle). At conjunction, a narrower, double-peaked line is formed at maximum displaced overall velocity due to motion along the cone.

Comparison of Fig. 1 and Fig. 2 shows how several interesting parameters can be extracted. First, the orbital inclination depends mainly on the maximum and minimum separation of the double-peaks: we find an inclination of $i \approx 63^{\circ}$ as a preliminary value for B22. The Lührs model also yields a 
shock-cone opening angle of $2 \beta \approx 94^{\circ}$. Finally, the cone material emitting $\mathrm{C}_{\text {III }} 5696 \AA$ is moving along the cone at a velocity of $v_{\mathrm{WR}} \approx 1570 \mathrm{~km} / \mathrm{s}$.

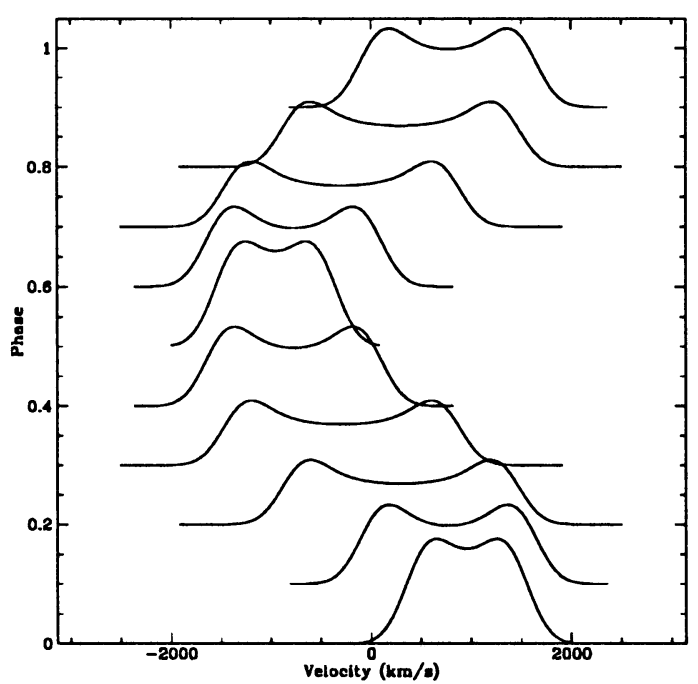

Fig. 2. Calculated profiles for a Lührs model of B22.

\section{Conclusion}

A spectroscopic study of all of the WC/WO stars of the Magellanic Clouds has shown very few obvious binary systems compared to the Galaxy, contrary to expectation.

As all of its $C_{\text {III }} 5696 \AA$ emission can be accounted for as excess emission from a wind shock, B22 should be considered as a non-emitter of Cir from its own wind and should be thus classified as WC4, rather than WC6.

The $\mathrm{C}_{\text {III }} 5696 \AA$ line profile is explained by a band of $\mathrm{C}_{\text {III }}$ produced downstream in the colliding wind bow shock. Other emission lines, such as $\mathrm{C}_{\mathrm{IV}}$, should be examined for profile changes.

\section{References}

Lührs, S. 1991, PhD thesis, Universität Münster

Maeder, A. 1991, $A \& A$ 242, 93

Moffat, A.F.J., Niemela, V.S., Marraco, H.G. 1990, ApJ 348, 232

Morgan, D.H., Good, A.R. 1985, MNRAS 216, 459

Smith, L.F., Shara, M.M., Moffat, A.F.J. 1990, $A p J$ 327, 128 


\section{DISCUSSION:}

Niemela: $\theta$ Mus seems very much alike B22 which you have studied. Have you tried to apply your model to $\theta$ Mus?

Bartzakos: I have not attempted to apply the model to $\theta$ Muscae. It is true that $\theta$ Muscae and B22 are similar. Moffat and Seggewiss studied this star in 1977 and the profile of CIII does vary like that of B22. It is interesting to note that both stars have similar periods. Perhaps the period of the system has some influence on whether or not such effects occur.

van der Hucht: Did you in your model consider spherical symmetry for the WR wind or a diskshaped wind? In case of the latter the collision cone around the $O$ star will also be asymmetric. Did you include this effect on modelling the line-shape variability?

Bartzakos: I assumed a spherically symmetric WR wind. The simplistic model can be thought of as a WR wind with the $\mathrm{O}$-star's wind blowing a hole in it. What I presented was made for demonstration purposes and proper calculations must follow.

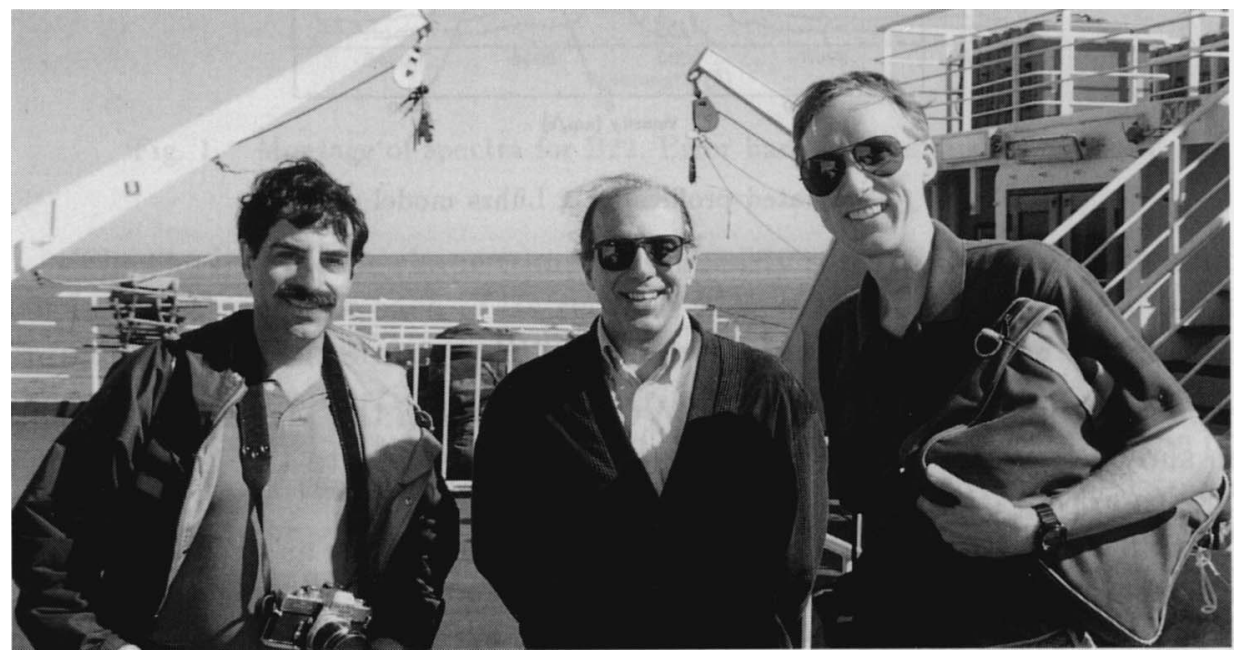

Cohen, Ferrini, Gies 